\title{
A perimeter enumeration of column-convex polyominoes
}

\author{
Svjetlan Feretić
}

Faculty of Civil Engineering, University of Rijeka, Viktora Cara Emina 5, 51000 Rijeka, Croatia

received Feb 27, 2006, revised April 24, 2007, accepted May 18, 2007.

\begin{abstract}
This work is concerned with the perimeter enumeration of column-convex polyominoes. We consider both the rectangular lattice and the hexagonal lattice. For the rectangular lattice, two formulae for the generating function already exist, and neither of them seems to admit further simplification. We first rederive those two formulae (so as to make the paper self-contained), and then we enrich the rectangular lattice generating function with some additional variables. Subsequently, we make a change of variables, which (nearly) produces the hexagonal lattice generating function. This latter generating function was first found by Lin and Wu in 1990. However, our present formula, in addition to having a simpler form, also allows a substantially easier Taylor series expansion. As to the methods, our approach is inspired by algebraic languages, whereas Lin and Wu used the Temperley methodology.
\end{abstract}

Keywords: Column-convex polyomino, Rectangular lattice, Hexagonal lattice, Perimeter generating function, Sequence of tailed polyominoes, Wall polyomino

\section{Introduction}

The enumeration of polyominoes (by perimeter and/or area) is a topic of great interest to physicists, chemists and combinatorialists alike. So far nobody has succeeded to count the set of all polyominoes. That set could easily remain beyond reach for a very long time. In view of this, it is easy to understand that those subsets of polyominoes which are reasonably numerous, but amenable to counting, enjoy considerable popularity. At present, not a wide variety of such subsets is known. In fact, each of today's numerous-yet-tractable sets of polyominoes is in some way derived either from column-convex polyominoes or from directed animals (or from both) (i) Column-convex polyominoes were first presented in Temperley's 1956 paper [15], and directed animals were first presented in Dhar, Phani and Barma's 1982 paper [5]. (It is plausible that directed animals appeared comparatively late because they do not submit to column-by-column approaches.)

On some lattices (such as e.g. the square lattice), directed animals have an algebraic area generating function, which satisfies a quadratic equation [1, 12, 14]. Moreover, stacked directed animals and multidirected animals (two recent models due to Bousquet-Mélou and Rechnitzer [2]) substantially generalize square-lattice directed animals and still have nice-looking area generating functions. On the other hand, the perimeter generating function for directed animals is not known, and is probably very complicated.

(i) In this paper, we somewhat imprecisely use the term "directed animal" for a directed site animal.

1365-8050 @ 2007 Discrete Mathematics and Theoretical Computer Science (DMTCS), Nancy, France 
Column-convex polyominoes have a rational area generating function and an algebraic perimeter generating function; the latter generating function satisfies a quartic equation. In detail, the cells of a columnconvex polyomino can be either squares or hexagons (other shapes are not so popular), but in both cases the generating functions have the nature just described.

Temperley [15] dealt with column-convex polyominoes with square cells. He computed the area generating function and started computing the perimeter generating function (which we denote $G_{s q}$ ), but he had to stop because the formulae became too bulky. Later on, Temperley's computations were completed by use of computer algebra systems. That was done in Brak, Guttmann and Enting [3], and in Lin [10]. Nevertheless, it was in Delest [4] that a formula for $G_{s q}$ was given for the first time; the papers [3, 10] appeared after [4]. Delest did not follow Temperley's approach. Instead, she used the Dyck-Schützenberger-Viennot method, a method in which polyominoes are encoded by words of algebraic languages. However, Delest's formula for $G_{s q}$ was very complicated. This is similar for the formulae of [3, 10]. (Our understanding is granted. Erdôs has stated that no one blames a mathematician if his or her first solution of a problem is messy [13, p. 289].) Later on, Feretić and Svrtan [8] recomputed $G_{s q}$ by the algebraic-language method, then upgraded the Temperley method, and then applied the upgraded method to derive $G_{s q}$ once again. As a result, in [8] there are two formulae for $G_{s q}$ (equivalent to one another), each of which is considerably simpler than the formulae of [3, 4, 10]. In the end, Feretić [6, 7] remarked that column-convex polyominoes are closely related to wall polyominoes of odd width. This remark allowed an easy rederivation of $G_{s q}$, and also accounted for the form which $G_{s q}$ has.

As to column-convex polyominoes with hexagonal cells, their area generating function was first found by Klarner [9], and their perimeter generating function (which we denote $G_{h e x}$ ) was first found by Lin and $\mathrm{Wu}$ [11]. Subsequently, Feretić and Svrtan [8] recomputed $G_{h e x}$ by two methods, just as they did with $G_{s q}$. So, in [8] there are two formulae for $G_{h e x}$, too. Each of these two formulae is shorter than Lin and Wu's formula for $G_{h e x}$. But, unfortunately, each of Feretić and Svrtan's [8] two formulae for $G_{h e x}$ is seriously wrong. (The moral is that every new formula needs a careful check.) Since [8] was published, no attempt has been made to derive $G_{h e x}$ once again. For a fairly long time, Lin and Wu's formula has remained the only—and hence the nicest—correct formula for $G_{h e x}$. In this paper, however, we shall express $G_{h e x}$ more simply and "Taylor-expandably" than Lin and Wu did. As to a check, we engaged Maple to expand our new expression for $G_{h e x}$, and then also Lin and Wu's expression for $G_{h e x}$, in a Taylor series through thirtieth-order terms. The two expansions agreed, and therefore we are convinced that our new formula is correct.

This paper continues as follows. In Section 2 , we state the necessary definitions. In Sections 3 to 5 , we repeat Feretić's [6] derivation of $G_{s q}$. (The repeated derivation appears in the present one as an essential part.) In particular, in Section 4 we find $K$, the perimeter generating function for wall polyominoes. In Section 6 , we enrich $K$ with an extra variable, which keeps track of repeats. In Section 7 , we digress to state the other formula for $G_{s q}$. In Section 8, we prove that the two formulae for $G_{s q}$ are equivalent. In Sections 9 to 12 , we study the properties of wall polyominoes of odd width. Each section is entitled after one or two properties therein considered. In Section 13 , wall polyominoes of odd width are already under our control, and we recast their generating function as a generating function for general column-convex polyominoes. In Section 14, a change of variables, together with a bit of gluing, gives us the series $G_{h e x}$. Finally, in Section 15, we outline a proof that the formula for $G_{h e x}$ found in Section 14 is equivalent to Lin and Wu's formula for $G_{h e x}$. 


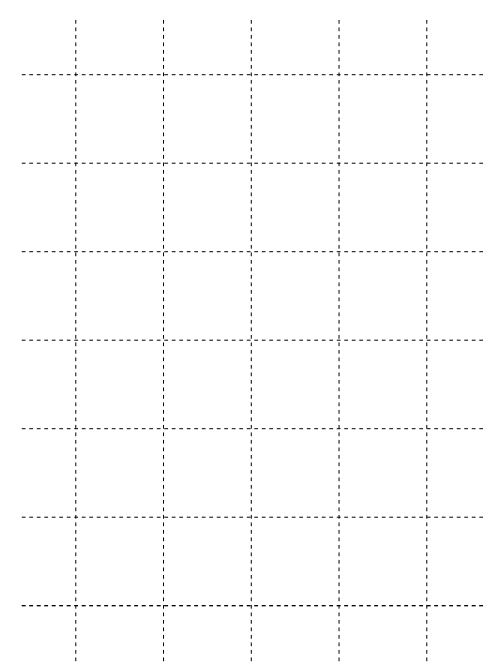

(a)

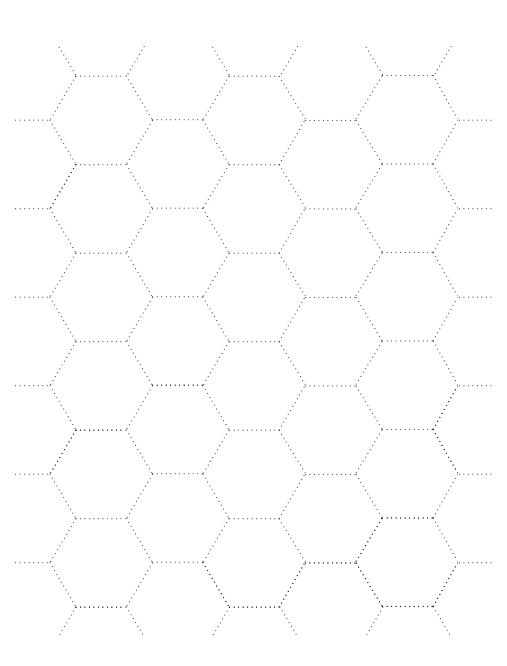

(b)

Fig. 1: (a) Square lattice and (b) hexagonal lattice.

\section{Annotated definitions}

A plane has three regular tilings: one with squares, one with hexagons, and one with triangles. The square, hexagonal and triangular tiles give rise to the square, hexagonal and triangular lattices, respectively. The hexagonal lattice is also known as the honeycomb lattice. In this paper, we confine our attention to the square and hexagonal lattices. We draw these two lattices so that every tile has two horizontal edges. See Fig. 1.

In a regularly tiled plane, a tile is often referred to as a cell. A polyomino is a finite union of cells which possesses a connected interior. A polyomino $P$ is column-convex if on the boundary of $P$ there are not more than two horizontal edges having the same horizontal projection. See Fig. 2

The cells of a column-convex polyomino $P$ fall into classes according to their horizontal projection. Each of those classes is called a column of $P$.

We denote the perimeter generating functions for column-convex polyominoes by $G_{s q}=G_{s q}(x, y)$ and $G_{h e x}=G_{h e x}(x, y, z)$. The series $G_{s q}$ refers to the square lattice. It is defined as follows:

$$
G_{s q}(x, y)=\sum_{\begin{array}{c}
P \text { a } \\
\text { column-convex } \\
\text { polyomino }
\end{array}} x^{\text {No. of horizontal edges of } P} \cdot y^{\text {No. of vertical edges of } P} .
$$

The series $G_{h e x}$ refers to the hexagonal lattice, and is defined by

$$
\begin{aligned}
& G_{\text {hex }}(x, y, z) \\
= & \sum_{\begin{array}{c}
P \text { a } \\
\text { column-convex } \\
\text { polyomino }
\end{array}} x^{\text {No. of horizontal edges of } P} \cdot y^{\text {No. of edges of } P \text { with a }+60^{\circ} \text { slope }} \cdot z^{\text {No. of edges of } P \text { with a }-60^{\circ} \text { slope }} .
\end{aligned}
$$




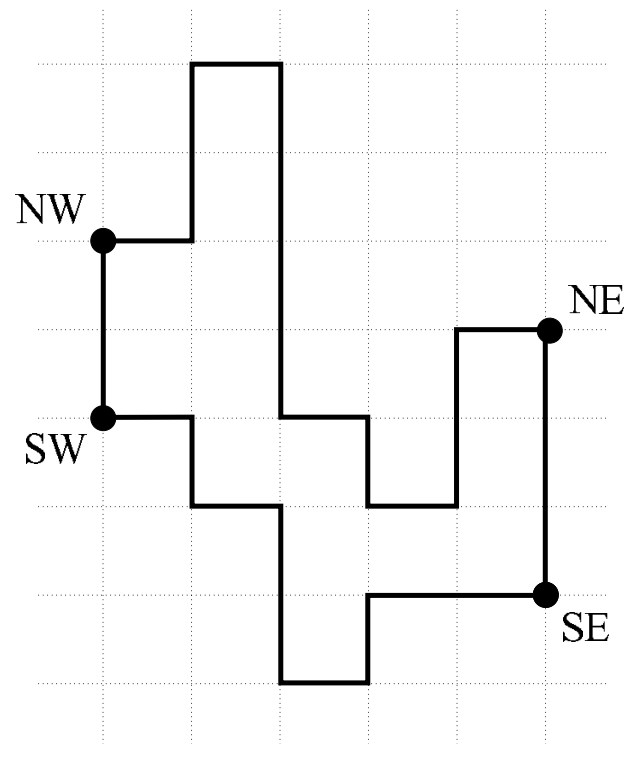

(a)

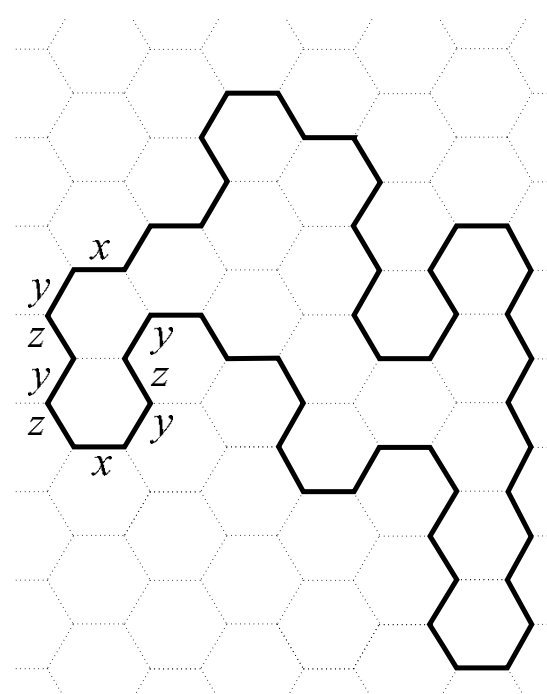

(b)

Fig. 2: Column-convex polyominoes on (a) the square lattice and (b) the hexagonal lattice. 


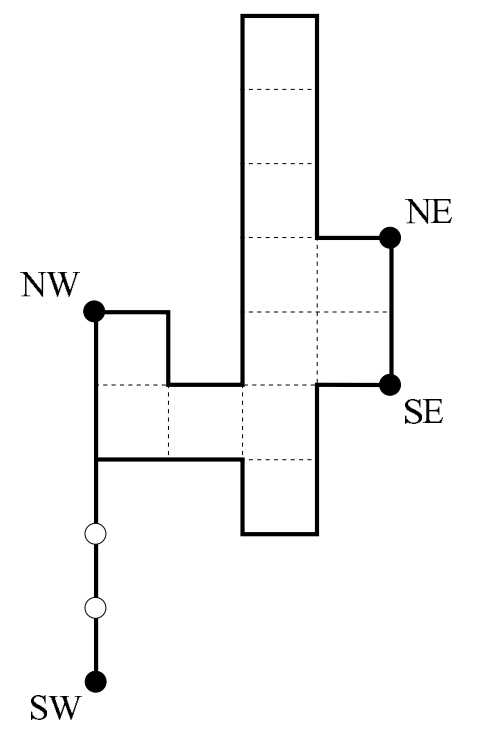

Fig. 3: A tapo.

In the rest of this section, as well as in the next ten sections, we concentrate on the square lattice.

For certain prominent points of a column-convex polyomino, we use the name poles and we write $S W, N W, N E$ and $S E$. For example, the $S W$ pole is the lower end of the left border of the leftmost column. Similarly for the other poles. See Fig. 2 again.

Imagine a column-convex polyomino $P$ with a "tail" $t$ of nonnegative integer length, going straight down from the $S W$ pole of $P$. We call the union $P \cup t$ a tailed polyomino or, briefly, a tapo. See Fig. 3 . The figure also shows the poles of a tapo: the $S W$ pole is the lower end of the tapo's tail, while the other three poles are inherited from the underlying column-convex polyomino.

For $n \in \mathbb{N}$, let $T_{1}$ be a column-convex polyomino, and let $T_{2}, T_{3}, \ldots, T_{n}$ be tapoes. Suppose that, for every $i \in\{2,3, \ldots, n\}$, the $S W$ pole of $T_{i}$ coincides with the $N E$ pole of $T_{i-1}$. Then we call the union $\bigcup_{i=1}^{n} T_{i}$ a stapo. (The name "stapo" is an abbreviation for a sequence of tailed polyominoes ${ }^{\text {(ii) }}$ ) Note that the stapoes with $n=1$ are simply column-convex polyominoes. See Fig. 4 .

We denote the perimeter generating functions for tapoes and stapoes by $H$ and $I$, respectively. Both $H$ and $I$ have two variables, $x$ and $y$. In the series $H$, the exponent of $x$ means the number of horizontal edges, and the exponent of $y$ in principle means the number of vertical edges. For computational convenience, however, we adopt the following agreement: if a tapo $T$ has a tail of length $k$, and the rest of $T$ is a column-convex polyomino with $2 i$ horizontal edges and $2 j$ vertical edges, then the contribution of $T$ to the series $H$ is $x^{2 i} y^{2 j+2 k}$ (and not $x^{2 i} y^{2 j+k}$ ). The series $I$ is defined likewise. That is, if a stapo $S$ has $2 i$ horizontal edges, $2 j$ vertical edges not belonging to tails, and $k$ vertical edges belonging to tails, then the contribution of $S$ to the series $I$ is again $x^{2 i} y^{2 j+2 k}$.

(ii) In the area of polyominoes there also exist stack polyominoes, close relatives of Ferrers diagrams. Hence, it should be stressed that "stapo" could be, but is not an abbreviation for "stack polyomino". 


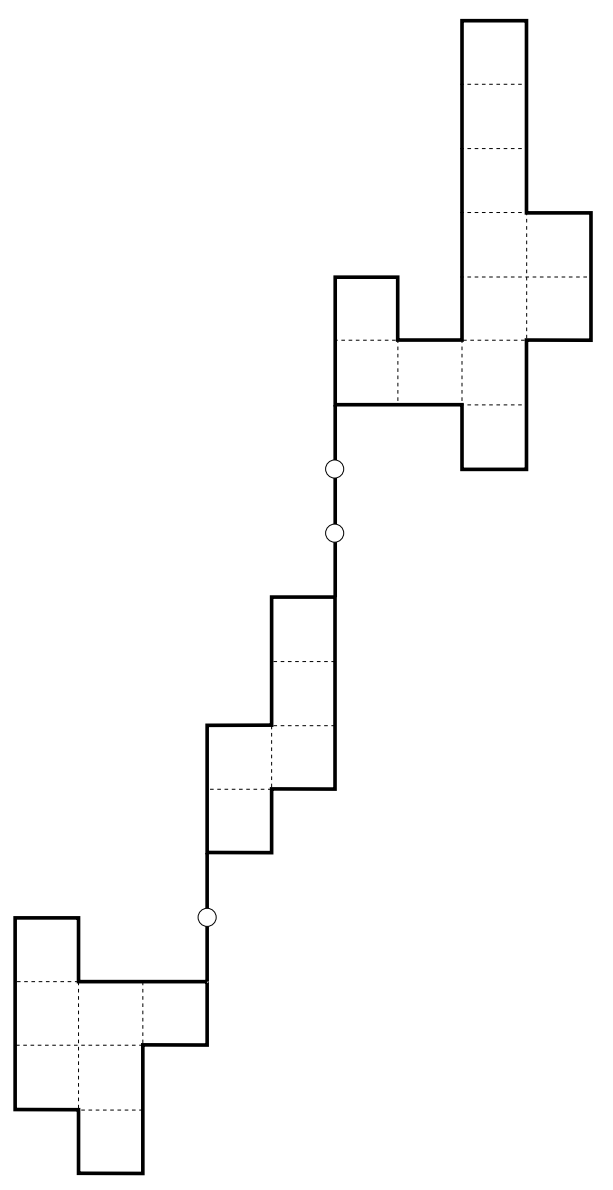

Fig. 4: A stapo. 
By virtue of the above definitions, we have

$$
H=G_{s q}\left(1+y^{2}+y^{4}+y^{6}+\ldots\right)=\frac{G_{s q}}{1-y^{2}}
$$

and

$$
I=G_{s q}\left(1+H+H^{2}+H^{3}+\ldots\right)=\frac{G_{s q}}{1-H} .
$$

Let $J=\frac{I}{1-y^{2}}$. We can think of $J$ as the perimeter generating function for objects which are very similar to stapoes, the only difference being that the leftmost column-convex polyomino, too, is allowed to have a tail. From (2) and (3) it follows that

$$
J=\frac{H}{1-H} .
$$

Hence

$$
H=1-\frac{1}{1+J} .
$$

A column-convex polyomino $P$ is a wall polyomino if the bottom sides of the columns of $P$ all lie on the same horizontal line. Wall polyominoes are also known as bargraph polygons.

We denote the perimeter generating function for wall polyominoes by $K=K(x, y)$. The definition of $K$ is

$$
K(x, y)=\sum_{P \text { a wall polyomino }} x^{\text {No. of columns of } P} \cdot y^{\text {No. of vertical edges of } P} .
$$

Thus, the roles of $x$ in $G_{s q}$ and in $K$ are somewhat different.

\section{A bijection between stapoes and wall polyominoes of odd width}

Stapoes are interesting because of a simple bijection which maps them onto the wall polyominoes of odd width. Let $S$ be a stapo with $n$ columns. Let $Y_{i}(S)$ and $y_{i}(S)$ be the maximal ordinate and the minimal ordinate of the $i$ th column of $S$. With $S$ we associate $\varphi(S)$, a wall polyomino having $2 n-1$ columns. From left to right, the heights of those columns are

$$
\begin{aligned}
Y_{1}(S)-y_{1}(S), \quad Y_{2}(S)-y_{1}(S), \quad Y_{2}(S)-y_{2}(S), \quad Y_{3}(S)-y_{2}(S), \\
\ldots, \quad Y_{n-1}(S)-y_{n-1}(S), \quad Y_{n}(S)-y_{n-1}(S), \quad \text { and } \quad Y_{n}(S)-y_{n}(S) .
\end{aligned}
$$

Thus, the 1st, 3rd, 5th, ... columns of the wall polyomino $\varphi(S)$ are copies of the 1st, $2 \mathrm{nd}, 3 \mathrm{rd}, \ldots$ columns of the stapo $S$. The 2 nd, 4 th, 6 th, $\ldots$ columns of $\varphi(S)$ tell us how the 2 nd, 3 rd, 4 th, $\ldots$ columns of $S$ are placed with respect to their left neighbour columns. For example, if the 6th column of $\varphi(S)$ is of height 5, then the top of the 4th column of $S$ lies 5 lattice units higher than the bottom of the 3rd column of $S$. See Fig. 5

With the above-stated agreement that a tail of length $k$ has $2 k$ vertical edges, the vertical perimeters of $S$ and $\varphi(S)$ relate in the simplest way. Namely, they are equal. On the other hand, the horizontal perimeter 


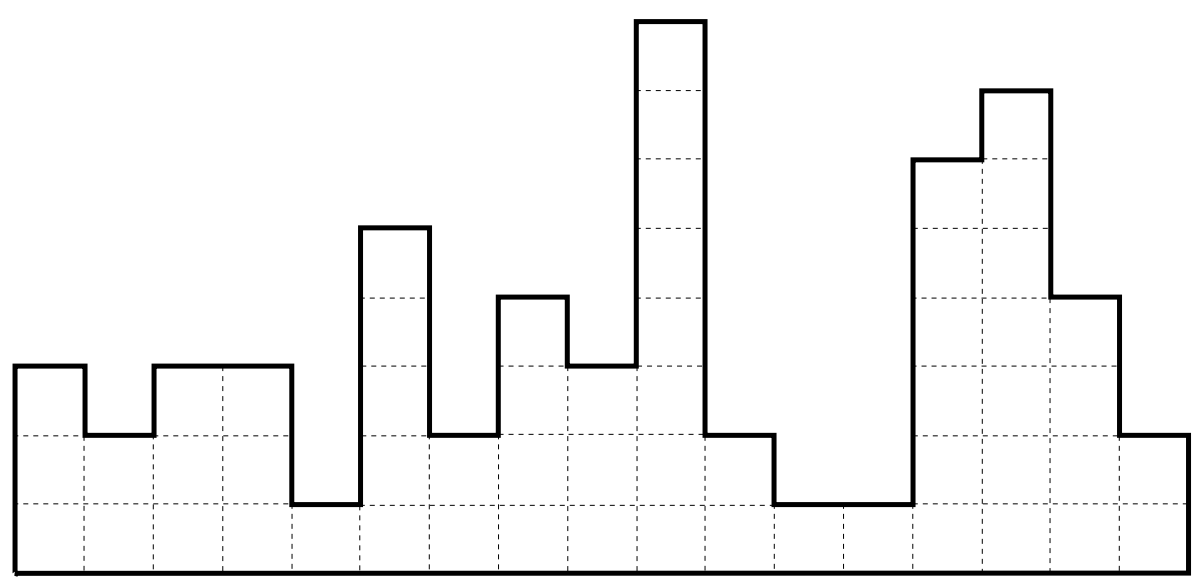

Fig. 5: Under the bijection $\varphi$, the stapo of Fig. 4 is mapped into this wall polyomino.

of $S$ is by one greater than the number of columns of $\varphi(S)$. So, the perimeter generating function for stapoes is $x$ times the perimeter generating function for wall polyominoes of odd width. That is,

$$
I=\frac{x}{2} \cdot[K(x, y)-K(-x, y)] .
$$

Now we shall compute $K$, and then it will be no problem to obtain $I, J, H$ and $G_{s q}$ one after another.

\section{The perimeter generating function for wall polyominoes}

Let $W$ denote the set of all wall polyominoes. Let $W_{\alpha}$ denote the set of wall polyominoes that have no one-celled columns. For $P \in W \backslash W_{\alpha}$, we define leone $(P)$ to be the leftmost one-celled column of $P$. We partition the set $W \backslash W_{\alpha}$ into four subsets: $W_{\beta}, W_{\gamma}, W_{\delta}$ and $W_{\epsilon}$. The definitions are:

- $W_{\beta}:=\left\{P \in W \backslash W_{\alpha}:\right.$ leone $(P)$ is both the leftmost and the rightmost column of $\left.P\right\}$,

- $W_{\gamma}:=\left\{P \in W \backslash W_{\alpha}:\right.$ leone $(P)$ is the leftmost, but not the rightmost column of $\left.P\right\}$,

- $W_{\delta}:=\left\{P \in W \backslash W_{\alpha}:\right.$ leone $(P)$ is the rightmost, but not the leftmost column of $\left.P\right\}$,

- $W_{\epsilon}:=\left\{P \in W \backslash W_{\alpha}:\right.$ leone $(P)$ is neither the leftmost nor the rightmost column of $\left.P\right\}$.

We write $K_{\alpha}, K_{\beta}, K_{\gamma}, K_{\delta}$ and $K_{\epsilon}$ to denote the parts of the series $K$ that come from the sets $W_{\alpha}, W_{\beta}$, $W_{\gamma}, W_{\delta}$ and $W_{\epsilon}$, respectively. With one eye on Fig. 6, we observe the following.

Between the sets $W$ and $W_{\alpha}$ there is an obvious bijection: Below a polyomino $P \in W$, we put a "pad" of height one. (The pad is as wide as $P$ is.) In this way, the vertical perimeter increases by two. Therefore, we have $K_{\alpha}=y^{2} K$.

The set $W_{\beta}$ has only one element, namely the one-celled polyomino. So, $K_{\beta}=x y^{2}$.

There also exists an obvious bijection between the sets $W$ and $W_{\gamma}$ : Just to the left of a polyomino $P \in W$, we place an extra column of height one. Under this bijection, the number of columns increases by one and the vertical perimeter remains the same. So, $K_{\gamma}=x K$. 

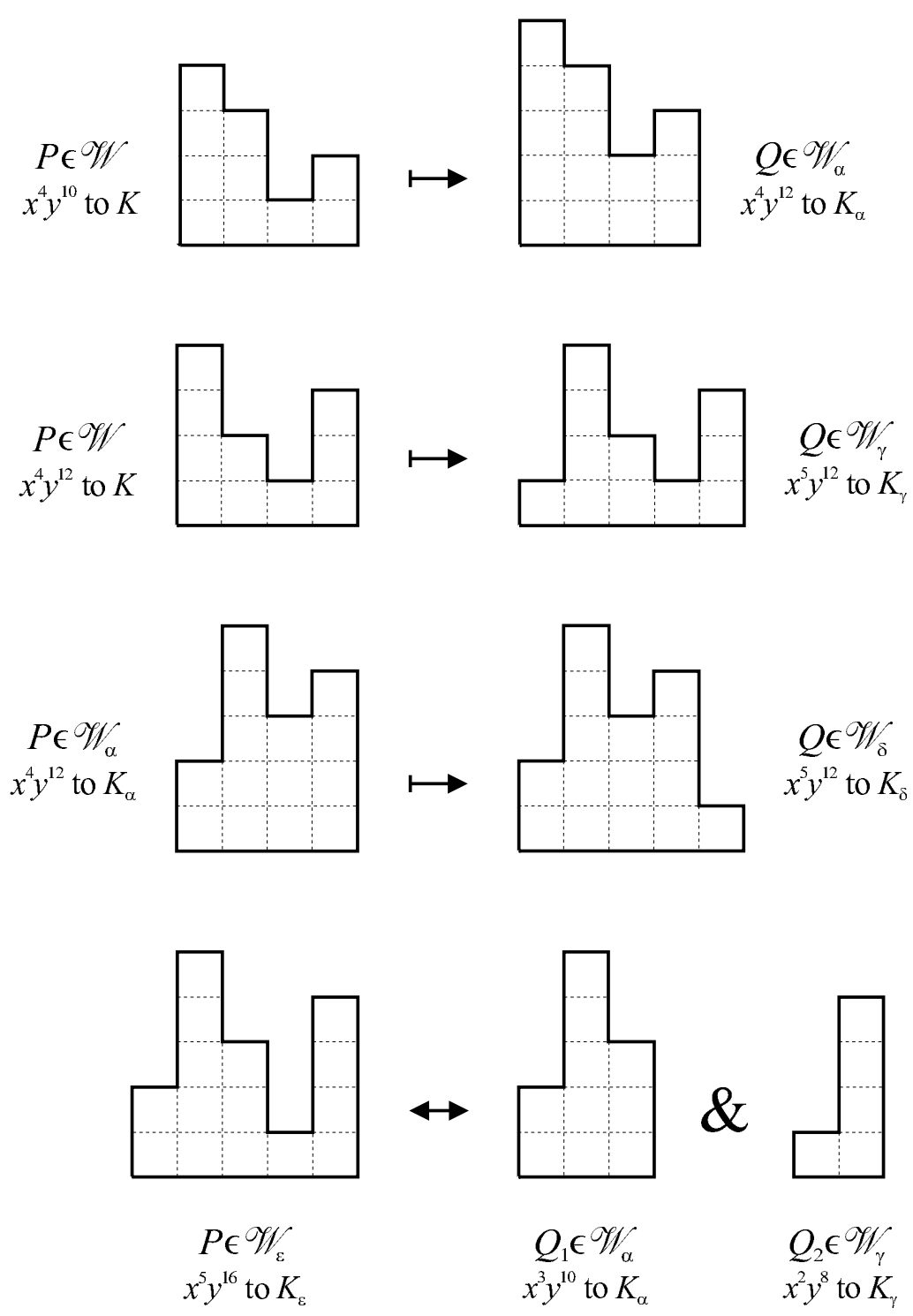

Fig. 6: The bijections used in establishing equation (7). 
There is yet another obvious bijection: Given $P \in W_{\alpha}$, we map it into $W_{\delta}$ by placing an extra column of height one just to the right of $P$. Under this bijection, too, the number of columns increases by one, while the vertical perimeter remains the same. So, $K_{\delta}=x K_{\alpha}=x y^{2} K$.

Finally, let $P \in W_{\epsilon}$. We decompose $P$ into two wall polyominoes, say $Q_{1}$ and $Q_{2}$. The polyomino $Q_{1}$ is a left factor; it begins with the first column of $P$ and ends with the column which is the left neighbour of leone $(P)$. Thus, $Q_{2}$ begins with leone $(P)$ and ends with the last column of $P$.

We see that $Q_{1}$ lies in $W_{\alpha}$ and that $Q_{2}$ lies in $W_{\gamma}$. The number of columns of $P$ is just the sum of the numbers of columns of $Q_{1}$ and $Q_{2}$. The vertical perimeter of $P$ is equal to vertical perimeter of $Q_{1}$ plus vertical perimeter of $Q_{2}$ minus two. Moreover, the decomposition $P \mapsto\left(Q_{1}, Q_{2}\right)$ is a bijection from $W_{\epsilon}$ to the Cartesian product $W_{\alpha} \times W_{\gamma}$. Altogether, we have $K_{\epsilon}=y^{-2} K_{\alpha} K_{\gamma}=x K^{2}$.

Since $K=K_{\alpha}+K_{\beta}+K_{\gamma}+K_{\delta}+K_{\epsilon}$, it follows that the generating function $K$ satisfies a quadratic equation:

$$
K=y^{2} K+x y^{2}+x K+x y^{2} K+x K^{2},
$$

which can be written as

$$
x K^{2}+\left[-\left(1-y^{2}\right)+x\left(1+y^{2}\right)\right] K+x y^{2}=0 .
$$

Solving equation (7), we obtain the following proposition.

Proposition 1 The perimeter generating function for wall polyominoes is given by

$$
K=\frac{1-y^{2}-x\left(1+y^{2}\right)-\sqrt{(1-x)^{2}\left(1-y^{2}\right)^{2}-4 x y^{2}\left(1-y^{2}\right)}}{2 x} .
$$

\section{A formula for $G_{s q}$}

By substituting (8) into (6), we get

$$
I=\frac{1}{4}\left[2\left(1-y^{2}\right)-\sqrt{(1-x)^{2}\left(1-y^{2}\right)^{2}-4 x y^{2}\left(1-y^{2}\right)}-\sqrt{(1+x)^{2}\left(1-y^{2}\right)^{2}+4 x y^{2}\left(1-y^{2}\right)}\right] .
$$

We defined $J$ to be $\frac{I}{1-y^{2}}$. Hence, we find

$$
J=\frac{2-\sqrt{(1-x)^{2}-\frac{4 x y^{2}}{1-y^{2}}}-\sqrt{(1+x)^{2}+\frac{4 x y^{2}}{1-y^{2}}}}{4} .
$$

By (2) and (5), $G_{s q}=\left(1-y^{2}\right) H=\left(1-y^{2}\right)\left(1-\frac{4}{4+4 J}\right)$. Here we insert (9), and the result reads as follows.

Theorem 1 The perimeter generating function for column-convex polyominoes on the rectangular lattice is given by

$$
G_{s q}=\left(1-y^{2}\right)\left[1-\frac{4}{6-\sqrt{(1-x)^{2}-\frac{4 x y^{2}}{1-y^{2}}}-\sqrt{(1+x)^{2}+\frac{4 x y^{2}}{1-y^{2}}}}\right] .
$$

Formula (10] first appeared in Feretić and Svrtan [8]. 


\section{Repeats}

Now that we know $G_{s q}$, we want to find $G_{h e x}$ as well. To achieve this goal, we are going to carry out a more detailed exploration of wall polyominoes. We shall take into account several new properties, the first of which will be repeats; by a repeat we mean the event that two neighbouring columns have the same height.

Let $L=L(x, y)$ be the part of $K$ that comes from wall polyominoes having no repeats. Let $M=$ $M(x, e, y)$ be defined by the formula

$$
M(x, e, y)=\sum_{P \text { a wall polyomino }} x^{\text {No. of columns of } P} \cdot e^{\text {No. of repeats of } P} \cdot y^{\text {No. of vertical edges of } P} .
$$

To see how the generating functions $K, L$ and $M$ relate to one another, it is useful to write down a procedure.

Procedure 1 Choose a wall polyomino $P$ without repeats. For every column $c$ of $P$, make either 0 , or 1 , or 2 , or $3, \ldots$ duplicates of $c$; then insert those duplicates just to the right of $c$.

The output of Procedure 1 is just $W$, the set of all wall polyominoes. Moreover, every wall polyomino is produced in a unique way. Thus, we have $M(x, e, y)=L\left(\frac{x}{1-x e}, y\right)$ and $L\left(\frac{x}{1-x}, y\right)=K(x, y)$. So,

$$
M(x, e, y)=L\left(\frac{x}{1-x e+x-x}, y\right)=L\left(\frac{\frac{x}{1-x e+x}}{1-\frac{x}{1-x e+x}}, y\right)=K\left(\frac{x}{1-x e+x}, y\right) .
$$

Therefore we substitute $\frac{x}{1-x e+x}$ for $x$ in 8 , and the result is

$$
M=\left\{(1-x e)\left(1-y^{2}\right)-2 x y^{2}-\sqrt{(1-x e)^{2}\left(1-y^{2}\right)^{2}-4 x y^{2}[1-x(e-1)]\left(1-y^{2}\right)}\right\} /(2 x) .
$$

To summarize, we changed a variable in a known generating function, and we obtained a generating function enriched with an extra variable. Later in this paper, this change-of-variable method will give us some results that seem hard to obtain in another way. In the case of formula (11), however, a simple alternative exists. It is quite possible to refine the proof of Proposition 1 so that repeats are taken into account, too. The reader may do this alternative proof by herself/himself.

Let

$$
M_{1}=\frac{M(x, e, y)-M(-x, e, y)}{2} .
$$

That is to say, $M_{1}$ is the portion of $M$ coming from wall polyominoes which have an odd number of columns. Using formula (11) and the notations

$$
\Delta_{-}=(1-x e)^{2}\left(1-y^{2}\right)^{2}-4 x y^{2}[1-x(e-1)]\left(1-y^{2}\right)
$$

and

$$
\Delta_{+}=(1+x e)^{2}\left(1-y^{2}\right)^{2}+4 x y^{2}[1+x(e-1)]\left(1-y^{2}\right),
$$

we readily obtain

$$
M_{1}=\frac{2\left(1-y^{2}\right)-\sqrt{\Delta_{-}}-\sqrt{\Delta_{+}}}{4 x} .
$$


Next we would like to have an algebraic equation for $M_{1}$. For this purpose, we first rewrite $(12)$ as

$$
\sqrt{\Delta_{+}}=2\left(1-y^{2}-2 x M_{1}\right)-\sqrt{\Delta_{-}} .
$$

By squaring and then rearranging, we get

$$
4 \sqrt{\Delta_{-}}\left(1-y^{2}-2 x M_{1}\right)=4\left(1-y^{2}-2 x M_{1}\right)^{2}+\Delta_{-}-\Delta_{+} .
$$

Then we square again, rearrange again, recall the definitions of $\Delta_{-}$and $\Delta_{+}$, and eventually divide the equation by $16\left(1-y^{2}\right)^{4}$. The result is

$$
\left(1-\frac{2 x M_{1}}{1-y^{2}}\right)^{4}-\left(1+x^{2} e^{2}+4 x^{2} y^{2} \frac{e-1}{1-y^{2}}\right)\left(1-\frac{2 x M_{1}}{1-y^{2}}\right)^{2}+x^{2}\left(e+\frac{2 y^{2}}{1-y^{2}}\right)^{2}=0 .
$$

\section{One more formula for $G_{s q}$}

The case $e=1$ of $(13)$ is also interesting. Let us turn to that case for a moment.

For $e=1, x M_{1}$ is $x[K(x, y)-K(-x, y)] / 2$, which is, by 6, equal to $I$. We defined $J$ to be $\frac{I}{1-y^{2}}$. Hence $\frac{x M_{1}}{1-y^{2}}$ is equal to $J$, and $J$ is, by (4), equal to $\frac{H}{1-H}$. So, from (13) we obtain

$$
\left(1-\frac{2 H}{1-H}\right)^{4}-\left(1+x^{2}\right)\left(1-\frac{2 H}{1-H}\right)^{2}+x^{2}\left(\frac{1+y^{2}}{1-y^{2}}\right)^{2}=0 .
$$

The solution of 14 is

$$
1-\frac{2 H}{1-H}=\frac{\sqrt{1+x^{2}+\sqrt{\left(1-x^{2}\right)^{2}-\frac{16 x^{2} y^{2}}{\left(1-y^{2}\right)^{2}}}}}{\sqrt{2}} .
$$

Since

$$
H=1-\frac{2}{3-\left(1-\frac{2 H}{1-H}\right)}
$$

it follows that

$$
H=1-\frac{2 \sqrt{2}}{3 \sqrt{2}-\sqrt{1+x^{2}+\sqrt{\left(1-x^{2}\right)^{2}-\frac{16 x^{2} y^{2}}{\left(1-y^{2}\right)^{2}}}}} .
$$

Multiplying by $1-y^{2}$, we obtain the following theorem.

Theorem 2 The perimeter generating function for column-convex polyominoes on the rectangular lattice is given by

$$
G_{s q}=\left(1-y^{2}\right)\left[1-\frac{2 \sqrt{2}}{3 \sqrt{2}-\sqrt{1+x^{2}+\sqrt{\left(1-x^{2}\right)^{2}-\frac{16 x^{2} y^{2}}{\left(1-y^{2}\right)^{2}}}}}\right] .
$$

Now we have two formulae for $G_{s q}$, viz. [10] and [15]. These formulae look rather different from one another, but they are of course equivalent. Both [10] and [15] were first obtained in [8]. 


\section{Proof that formulae (10) and (15) are equivalent}

Under the inner square root in 15 , we do a series of rewritings:

$$
\begin{aligned}
\left(1-x^{2}\right)^{2}-\frac{16 x^{2} y^{2}}{\left(1-y^{2}\right)^{2}} & =\left(1+x^{2}\right)^{2}-\frac{4 x^{2}\left(1+y^{2}\right)^{2}}{\left(1-y^{2}\right)^{2}} \\
& =\left[1+x^{2}-\frac{2 x\left(1+y^{2}\right)}{1-y^{2}}\right]\left[1+x^{2}+\frac{2 x\left(1+y^{2}\right)}{1-y^{2}}\right] \\
& =\left[(1-x)^{2}-\frac{4 x y^{2}}{1-y^{2}}\right]\left[(1+x)^{2}+\frac{4 x y^{2}}{1-y^{2}}\right] .
\end{aligned}
$$

Using this, we rewrite the contents of the outer square root as follows:

$$
\begin{aligned}
1+x^{2}+\sqrt{\left(1-x^{2}\right)^{2}-\frac{16 x^{2} y^{2}}{\left(1-y^{2}\right)^{2}}} & =\frac{1}{2}\left[(1-x)^{2}-\frac{4 x y^{2}}{1-y^{2}}+2 \sqrt{(1-x)^{2}-\frac{4 x y^{2}}{1-y^{2}} \sqrt{(1+x)^{2}+\frac{4 x y^{2}}{1-y^{2}}}}+(1+x)^{2}+\frac{4 x y^{2}}{1-y^{2}}\right] \\
& =\frac{1}{2}\left[\sqrt{(1-x)^{2}-\frac{4 x y^{2}}{1-y^{2}}}+\sqrt{(1+x)^{2}+\frac{4 x y^{2}}{1-y^{2}}}\right]^{2} .
\end{aligned}
$$

Thus, the big fraction in 15 is equal to

$$
\frac{2 \sqrt{2}}{3 \sqrt{2}-\frac{1}{\sqrt{2}}\left[\sqrt{(1-x)^{2}-\frac{4 x y^{2}}{1-y^{2}}}+\sqrt{(1+x)^{2}+\frac{4 x y^{2}}{1-y^{2}}}\right]}=\frac{4}{6-\sqrt{(1-x)^{2}-\frac{4 x y^{2}}{1-y^{2}}}-\sqrt{(1+x)^{2}+\frac{4 x y^{2}}{1-y^{2}}}}
$$

which is identical to the big fraction in 10$]$. Therefore, (15) can be rearranged into 10 , and vice versa.

\section{OEO repeats and single repeats}

The digression finished, the variable $e$ is around again.

By substituting $\sqrt{x}$ for $x$ in the series $x M_{1}$, we obtain a new series, say $N$. We have

$$
N(x, e, y)=\sum_{\substack{P \text { a wall polyomino } \\ \text { of odd width }}} x^{\text {No. of odd columns of } P} \cdot e^{\text {No. of repeats of } P} \cdot y^{\text {vertical perimeter of } P} .
$$

Here, it is understood that the odd columns of a wall polyomino are its first, third, fifth, ... columns, while the even columns are those columns which are not odd.

From (13) it follows that $N$ satisfies the equation

$$
\left(1-\frac{2 N}{1-y^{2}}\right)^{4}-\left(1+x e^{2}+4 x y^{2} \frac{e-1}{1-y^{2}}\right)\left(1-\frac{2 N}{1-y^{2}}\right)^{2}+x\left(e+\frac{2 y^{2}}{1-y^{2}}\right)^{2}=0
$$


The next property of interest are the OEO repeats (iii). By definition, an OEO repeat is the event that three successive columns have the same height, and the first of those three columns is an odd column. We also define single repeats: A single repeat is a repeat not forming part of any OEO repeat.

Let

$$
\begin{array}{ll}
O(x, d, e, y)=\sum_{\begin{array}{c}
P \text { a wall polyomino } \\
\text { of odd width }
\end{array}} & x^{\text {No. of odd columns of } P} \cdot d^{\text {No. of } O E O \text { repeats of } P} \\
& \cdot e^{\text {No. of single repeats of } P} \cdot y^{\text {vertical perimeter of } P} .
\end{array}
$$

Then $O(x, 0, e, y)$ is the part of $N$ that comes from wall polyominoes of odd width having no OEO repeats. To see how the generating functions $N, O(x, 0, e, y)$ and $O(x, d, e, y)$ relate to one another, it is useful to write down a procedure.

Procedure 2 Choose a wall polyomino of odd width having no OEO repeats. In the chosen polyomino, focus on the odd columns. For every such column c, make either 0 , or 2 , or 4, or $6, \ldots$ duplicates of c; then insert those duplicates just to the right of $c$.

The output of Procedure 2 is just the set of all wall polyominoes of odd width. Moreover, every wall polyomino of odd width is produced in a unique way. Thus, we have $O(x, d, e, y)=O\left(\frac{x}{1-x d}, 0, e, y\right)$ and $O\left(\frac{x}{1-x e^{2}}, 0, e, y\right)=N(x, e, y)$. Hence,

$$
\begin{aligned}
O(x, d, e, y) & =O\left(\frac{x}{1+x\left(e^{2}-d\right)-x e^{2}}, 0, e, y\right) \\
& =O\left(\frac{\frac{x}{1+x\left(e^{2}-d\right)}}{1-\frac{x}{1+x\left(e^{2}-d\right)} e^{2}}, 0, e, y\right) \\
& =N\left(\frac{x}{1+x\left(e^{2}-d\right)}, e, y\right)
\end{aligned}
$$

\section{Low and high single repeats}

Now that we drew a distinction between OEO repeats and single repeats, we wish to draw a further distinction between two kinds of single repeats. The definitions follow.

Every single repeat involves one even column, which is equally high as one (but not both) of its neighbour columns. If the even column is lower than its unlike neighbour column, we speak of a low single repeat. Otherwise we speak of a high single repeat.

Given a low single repeat, we can of course heighten the even column involved so as to produce a high single repeat. The point is that this low-to-high conversion does not affect the vertical perimeter. See Fig. 7. Naturally, under the similar conversion from a high single repeat into a low single repeat, the vertical perimeter is again unaffected.

For $P$ a wall polyomino of odd width, we define a companion of $P$ to be a wall polyomino which can be obtained from $P$ by means of low-to-high and high-to-low conversions. We also define LSR polyominoes: An LSR polyomino is a wall polyomino of odd width which has no high single repeats. Every wall polyomino of odd width is a companion to one and only one LSR polyomino.

(iii) OEO is an abbreviation for odd-even-odd. 

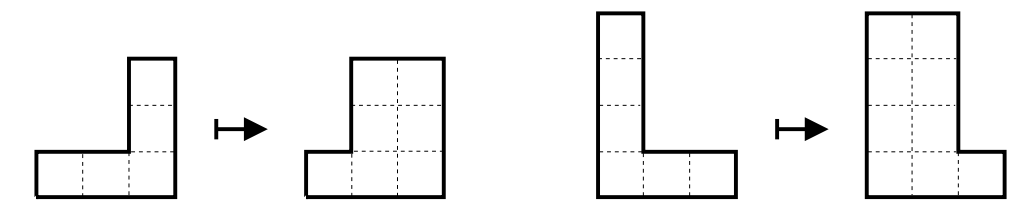

$$
x^{2} d^{0} e^{1} y^{6} \text { to } O \quad x^{2} d^{0} e^{1} y^{6} \text { to } O \quad x^{2} d^{0} e^{1} y^{8} \text { to } O \quad x^{2} d^{0} e^{1} y^{8} \text { to } O
$$

Fig. 7: The low-to-high conversion.

Let

$$
\begin{gathered}
P\left(x, a_{0}, b_{0}, d, y\right)=\sum_{\begin{array}{c}
W \text { a wall } \\
\text { polyomino } \\
\text { of odd width }
\end{array}} x^{\text {No. of odd columns of } W} \cdot a_{0}^{\text {No. of high single repeats of } W} \\
\cdot b_{0}^{\text {No. of low single repeats of } W} \cdot d^{\text {No. of } O E O \text { repeats of } W} \cdot y^{\text {vertical perimeter of } W} .
\end{gathered}
$$

Then $P\left(x, 0, b_{0}, d, y\right)$ is a generating function for LSR polyominoes. An LSR polyomino has the same vertical perimeter as any of its companions. Hence $P\left(x, 0, a_{0}+b_{0}, d, y\right)$ is a generating function for LSR polyominoes and all of their companions or, in other words, a generating function for all wall polyominoes of odd width. Thus, $P\left(x, a_{0}, b_{0}, d, y\right)=P\left(x, 0, a_{0}+b_{0}, d, y\right)$. Since $P(x, e, e, d, y)=$ $O(x, d, e, y)$, it follows that $P\left(x, a_{0}, b_{0}, d, y\right)=P\left(x, 0, a_{0}+b_{0}, d, y\right)=P\left(x, 0, \frac{a_{0}+b_{0}}{2}+\frac{a_{0}+b_{0}}{2}, d, y\right)$ $=P\left(x, \frac{a_{0}+b_{0}}{2}, \frac{a_{0}+b_{0}}{2}, d, y\right)=O\left(x, d, \frac{a_{0}+b_{0}}{2}, y\right)$.

Now we use relation (17) to obtain

$$
P\left(x, a_{0}, b_{0}, d, y\right)=N\left(\frac{x}{1+x\left[\frac{\left(a_{0}+b_{0}\right)^{2}}{4}-d\right]}, \frac{a_{0}+b_{0}}{2}, y\right)
$$

\section{Chimneys}

Having beaten the even columns which play a role in repeats, we now turn to the even columns which play no role in repeats.

We define a chimney to be an even column which is higher than both of its neighbour columns.

Mutatis mutandi, we handle chimneys as we handled repeats and OEO repeats. That is, first we define a generating function and then we write down a procedure.

Let

$$
\begin{aligned}
Q\left(x, a_{0}, a, b_{0}, d, y\right)=\sum_{\begin{array}{c}
P \text { a wall } \\
\text { polyomino } \\
\text { of odd width }
\end{array}} & x^{\text {No. of odd columns of } P} \cdot a_{0}^{\text {No. of high single repeats of } P} \\
& \cdot a^{\text {No. of chimneys of } P} \cdot b_{0}^{\text {No. of low single repeats of } P} \\
& \cdot d^{\text {No. of } O E O \text { repeats of } P} y^{\text {vertical perimeter of } P} .
\end{aligned}
$$

Then $Q\left(x, a_{0}, 0, b_{0}, d, y\right)$ is the part of the generating function $P$ that comes from wall polyominoes of odd width having no chimneys. 

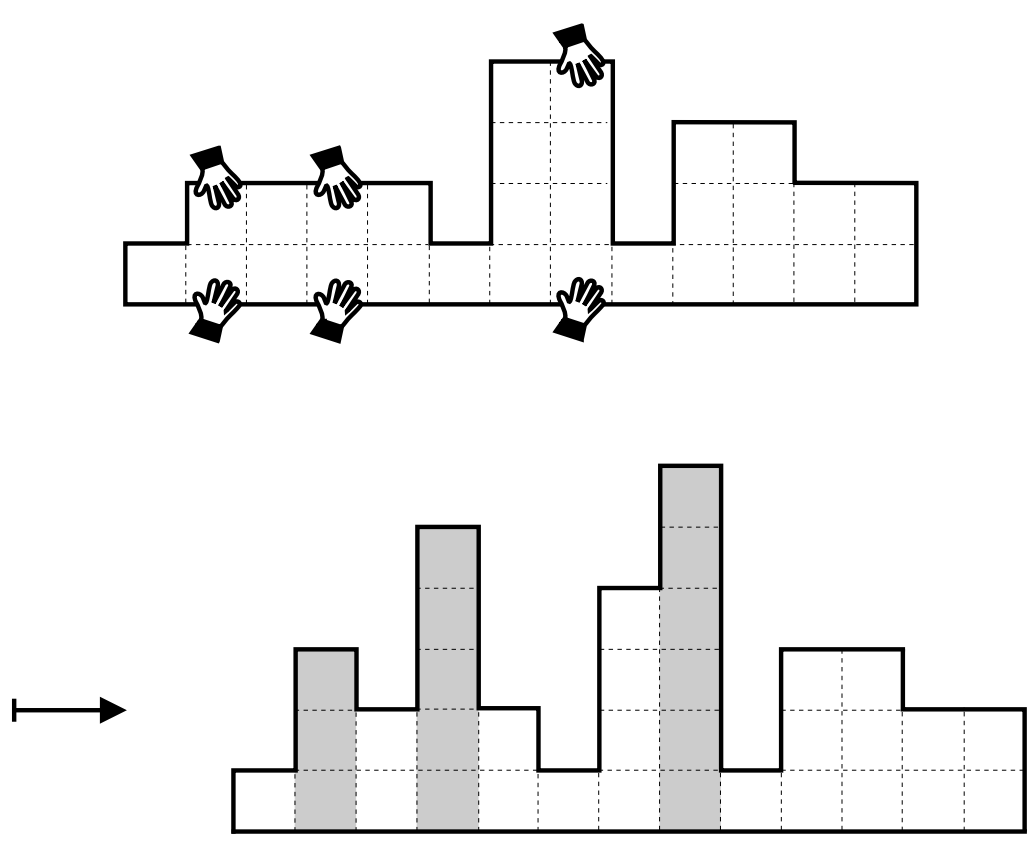

Fig. 8: Making of chimneys.

\section{Procedure 3}

- Choose a wall polyomino of odd width having no chimneys.

- In the chosen wall polyomino, choose some (or none) of the even columns living in high single repeats. Also choose some (or none) of the even columns living in OEO repeats.

- Visit the chosen columns one by one, each time placing either 1 , or 2 , or $3, \ldots$ additional cells on the top of the column being visited.

This procedure is illustrated in Fig. 8

The output of Procedure 3 is just the set of all wall polyominoes of odd width. Moreover, every wall polyomino of odd width is produced in a unique way. Thus, we have $Q\left(x, a_{0}, a, b_{0}, d, y\right)=Q\left(x, a_{0}+\right.$ $\left.\frac{a y^{2}}{1-y^{2}}, 0, b_{0}, d+\frac{a y^{2}}{1-y^{2}}, y\right)$ and $Q\left(x, a_{0}+\frac{y^{2}}{1-y^{2}}, 0, b_{0}, d+\frac{y^{2}}{1-y^{2}}, y\right)=P\left(x, a_{0}, b_{0}, d, y\right)$. Therefore

$$
\begin{aligned}
Q\left(x, a_{0}, a, b_{0}, d, y\right) & =Q\left(x, a_{0}+\frac{(a-1) y^{2}}{1-y^{2}}+\frac{y^{2}}{1-y^{2}}, 0, b_{0}, d+\frac{(a-1) y^{2}}{1-y^{2}}+\frac{y^{2}}{1-y^{2}}, y\right) \\
& =P\left(x, a_{0}+\frac{(a-1) y^{2}}{1-y^{2}}, b_{0}, d+\frac{(a-1) y^{2}}{1-y^{2}}, y\right) .
\end{aligned}
$$


With this relation in hand, we use (18) to obtain

$$
Q\left(x, a_{0}, a, b_{0}, d, y\right)=N\left(\frac{x}{1+x\left\{\frac{\left[a_{0}+\frac{(a-1) y^{2}}{1-y^{2}}+b_{0}\right]^{2}}{4}-d-\frac{(a-1) y^{2}}{1-y^{2}}\right\}}, \frac{a_{0}+\frac{(a-1) y^{2}}{1-y^{2}}+b_{0}}{2}, y\right) .
$$

\section{Wells}

We are now ready for the next (and last) property.

A well is an even column which is lower than both of its neighbour columns.

Wells are somewhat trickier than chimneys. To conquer wells, it is not enough to make a change of variables. So to speak, it is also necessary to make a change of the generating function. Anyway, the job begins in the usual way: first we define a generating function and then we write down a procedure.

Let

$$
\begin{aligned}
R\left(x, a_{0}, a, b_{0}, b, d, y\right)=\sum_{\begin{array}{c}
P \text { a wall } \\
\text { polyomino } \\
\text { of odd width }
\end{array}} & x^{\text {No. of odd columns of } P} \\
& \cdot a_{0}^{\text {No. of high single repeats of } P} \cdot a^{\text {No. of chimneys of } P} \\
& \cdot b_{0}^{\text {No. of low single repeats of } P} \cdot b^{\text {No. of wells of } P} \\
& \cdot d^{\text {No. of } O E O \text { repeats of } P} \cdot y^{\text {vertical perimeter of } P} .
\end{aligned}
$$

Then $R\left(x, a_{0}, a, b_{0}, 0, d, y\right)$ is the part of $Q$ that comes from wall polyominoes of odd width having no wells.

\section{Procedure 4}

- Choose a wall polyomino of odd width having no wells.

- In the chosen wall polyomino, choose some (or none) of the even columns living in low single repeats. Also choose some (or none) of the even columns living in OEO repeats.

- Visit the chosen columns one by one, each time lowering the column being visited either by 1 , or by 2 , or by $3, \ldots$.

This procedure is illustrated in Fig. 9

Notice that Procedure 4 generates polyominoes and non-polyominoes alike. That happens because when we lower a column, we are free to overdraw. For example, from a column of height 3 , we get an (ex-)column whose height may be any of the numbers $2,1,0,-1,-2,-3, \ldots$ Thus, the output of Procedure 4 is a relatively large set: it contains every object whose form is either $\sqcap$ or $\sqcap \sqcup \sqcap$ or $\sqcap \sqcup \sqcap \sqcup \sqcap$ or .... Here, every $\sqcap$ is a wall polyomino of odd width, and every $\sqcup$ is an ex-column of nonpositive height.

These facts observed, what can we say about generating functions? After a little thought, we realize that

$$
\frac{R\left(x, a_{0}, a, b_{0}, b, d, y\right)}{1-\frac{b R\left(x, a_{0}, a, b_{0}, b, d, y\right)}{1-y^{2}}}=R\left(x, a_{0}, a, b_{0}+\frac{b y^{2}}{1-y^{2}}, 0, d+\frac{b y^{2}}{1-y^{2}}, y\right)
$$



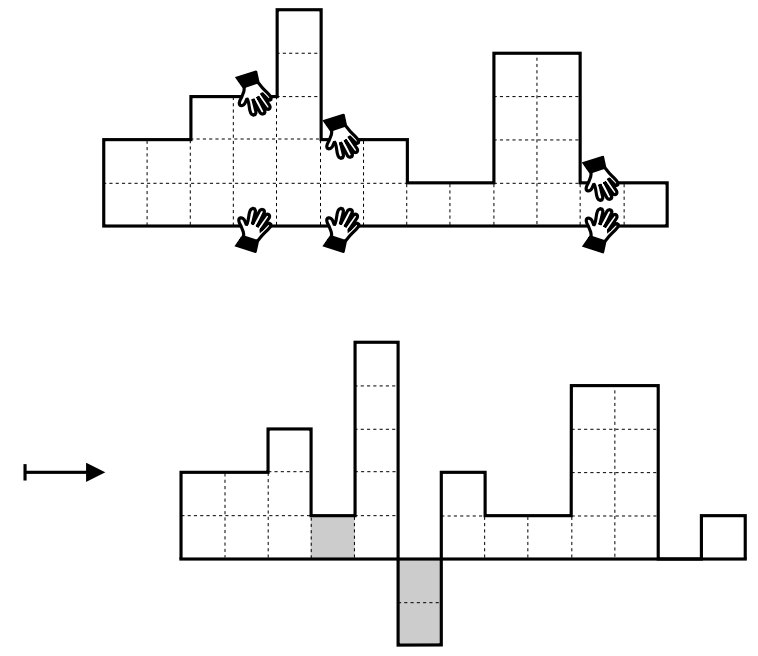

Fig. 9: Making of wells, with the freedom to overdraw.

and that

$$
R\left(x, a_{0}, a, b_{0}+\frac{y^{2}}{1-y^{2}}, 0, d+\frac{y^{2}}{1-y^{2}}, y\right)=\frac{Q\left(x, a_{0}, a, b_{0}, d, y\right)}{1-\frac{Q\left(x, a_{0}, a, b_{0}, d, y\right)}{1-y^{2}}}
$$

Hence,

$$
\begin{aligned}
\frac{R\left(x, a_{0}, a, b_{0}, b, d, y\right)}{1-\frac{b R\left(x, a_{0}, a, b_{0}, b, d, y\right)}{1-y^{2}}} & =R\left(x, a_{0}, a, b_{0}+\frac{(b-1) y^{2}}{1-y^{2}}+\frac{y^{2}}{1-y^{2}}, 0, d+\frac{(b-1) y^{2}}{1-y^{2}}+\frac{y^{2}}{1-y^{2}}, y\right) \\
& =\frac{Q\left(x, a_{0}, a, b_{0}+\frac{(b-1) y^{2}}{1-y^{2}}, d+\frac{(b-1) y^{2}}{1-y^{2}}, y\right)}{1-\frac{Q\left(x, a_{0}, a, b_{0}+\frac{(b-1) y^{2}}{1-y^{2}}, d+\frac{(b-1) y^{2}}{1-y^{2}}, y\right)}{1-y^{2}}},
\end{aligned}
$$

which means that

$$
\frac{\left(1-y^{2}\right) R}{1-y^{2}-(b-1) R}=Q\left(x, a_{0}, a, b_{0}+\frac{(b-1) y^{2}}{1-y^{2}}, d+\frac{(b-1) y^{2}}{1-y^{2}}, y\right) .
$$

Combining (20) with (19), we get the following result:

$$
\frac{\left(1-y^{2}\right) R}{1-y^{2}-(b-1) R}=N\left(\frac{x}{1+x\left\{\frac{\left[a_{0}+b_{0}+\frac{(a+b-2) y^{2}}{1-y^{2}}\right]^{2}}{4}-d-\frac{(a+b-2) y^{2}}{1-y^{2}}\right\}}, \frac{a_{0}+b_{0}+\frac{(a+b-2) y^{2}}{1-y^{2}}}{2}, y\right) .
$$




\section{The twilight of wall polyominoes}

Our additional variables $a_{0}, a, b_{0}, b$ and $d$ have done us very good service. At this stage, however, it is possible to reduce the set of variables without incurring a serious loss of information.

From $R\left(x, a_{0}, a, b_{0}, b, d, y\right)$, a generating function with seven variables, we pass to a generating function with four variables. Let

$$
\begin{aligned}
S\left(x, a, b_{0}, y\right)= & \sum_{\begin{array}{c}
P \text { a wall } \\
\text { polyomino } \\
\text { of odd width }
\end{array}} x^{\text {No. of columns plus one of } P} \cdot a^{\text {No. of chimneys of } P} \\
& \cdot b_{0}^{\text {total No. of low single repeats, wells and } O E O \text { repeats of } P} \cdot y^{\text {vertical perimeter of } P} .
\end{aligned}
$$

Noticing that $S=R\left(x^{2}, 1, a, b_{0}, b_{0}, b_{0}, y\right)$, from (21) we get

$$
\frac{\left(1-y^{2}\right) S}{1-y^{2}-\left(b_{0}-1\right) S}=N\left(\frac{x^{2}}{1+x^{2} \lambda_{-}^{2}}, 1+\lambda_{-}, y\right)
$$

where

$$
\lambda_{-}=\frac{(a-1) y^{2}+b_{0}-1}{2\left(1-y^{2}\right)} .
$$

Recall that in Section 3 we defined a bijection $\varphi$, which took us from stapoes to wall polyominoes of odd width. Now is the time to go back to stapoes. As a means of transportation, we shall use the bijection $\varphi^{-1}$. So, which kind of generating function for stapoes is the series $S$ ? Before answering this question, it is useful to give names to some properties of a stapo.

When we speak about a column of a stapo, and no ambiguity need be feared, we denote the maximum ordinate and the minimum ordinate of that column by $Y$ and $y$, respectively.

Let a rise be the event that, in a pair of adjacent columns of a stapo, $Y$ of the right column is greater than $Y$ of the left column, and $y$ of the right column is greater than $y$ of the left column. Let a weak fall be the event that, in a pair of adjacent columns of a stapo, $Y$ of the right column is less than or equal to $Y$ of the left column, and $y$ of the right column is less than or equal to $y$ of the left column.

Now, the alter ego of the series $S$ is

$$
\begin{aligned}
S=\sum_{P \text { a stapo }} & x^{\text {horizontal perimeter of } P} \cdot a^{\text {No. of rises of } P} \\
& \cdot b_{0}^{\text {No. of weak falls of } P} \cdot y^{\text {vertical perimeter of } P} .
\end{aligned}
$$

Next we define a generating function for tapoes and a generating function for column-convex polyominoes. The names of these generating functions are $T=T\left(x, a, b_{0}, y\right)$ and $G_{s q+}=G_{s q+}\left(x, a, b_{0}, y\right)$, respectively. In each of the generating functions $T$ and $G_{s q+}$, each of the variables has the same meaning as in $S$.

We have

$$
S=G_{s q+}(1+a T+a T a T+a T a T a T+\ldots)=\frac{G_{s q+}}{1-a T}
$$


Also, $T=G_{s q+}\left(1+y^{2}+y^{4}+y^{6}+\ldots\right)=\frac{G_{s q+}}{1-y^{2}}$, so that $\left(1-y^{2}\right) T=G_{s q+}$. Therefore

$$
\begin{aligned}
\frac{\left(1-y^{2}\right) S}{1-y^{2}-\left(b_{0}-1\right) S} & =\frac{\left(1-y^{2}\right) \frac{G_{s q+}}{1-a T}}{1-y^{2}-\left(b_{0}-1\right) \frac{G_{s q+}}{1-a T}} \\
& =\frac{\left(1-y^{2}\right) G_{s q+}}{\left(1-y^{2}\right)(1-a T)-\left(b_{0}-1\right) G_{s q+}} \\
& =\frac{\left(1-y^{2}\right) G_{s q+}}{1-y^{2}-\left(a+b_{0}-1\right) G_{s q+}} .
\end{aligned}
$$

Substitution of this into 22] yields

$$
\frac{\left(1-y^{2}\right) G_{s q+}}{1-y^{2}-\left(a+b_{0}-1\right) G_{s q+}}=N\left(\frac{x^{2}}{1+x^{2} \lambda_{-}^{2}}, 1+\lambda_{-}, y\right),
$$

with $\lambda_{-}$still given by 23$]$.

\section{Coping with the honeycomb lattice}

Next, since our business is expanding again, we employ a new variable $z$. That is to say, we pass to the generating function $G_{s q+}\left(x, a, b_{0}, y z\right)$, where we think of $y$ and $z$ as the variables counting the first halves and second halves of vertical edges, respectively. Here, "the first half" actually means the half that would be first if the boundary of a column-convex polyomino had anticlockwise orientation.

Then we subject every column-convex polyomino $P$ to a series of half-a-unit downward shifts. First we shift all of $P$ except for the leftmost column of $P$. Second we shift all of the first-shifted object, except for the leftmost column of that object. Third we shift all of the second-shifted object, except for the leftmost column of that object. And so on. In the last shift, the shifted object has only one column.

Through this series of shifts, a column-convex polyomino becomes similar to a piece of a brick wall. See Fig. 10 .

To count those (so to speak) pieces of brick walls, we introduce a generating function $U=U(x, y, z)$. The definition is

$$
\begin{gathered}
U(x, y, z)=\sum_{\begin{array}{c}
P \text { a piece } \\
\text { of a brick wall }
\end{array}} x^{\text {horizontal perimeter of } P} \\
\cdot y^{\text {No. of first halves of vertical edges of } P} \cdot z^{\text {No. of second halves of vertical edges of } P} .
\end{gathered}
$$

(The meaning of "the first half" and "the second half" is illustrated in Fig. 11.) Of course, our aim is to express $U$ by means of $G_{s q+}$. So, how do the shifts influence the vertical perimeter? By taking a closer look at Fig. 11] we find out the following.

A shift has influence in just two cases. Case 1: When the overlap between the moving columns and the still columns is a rise, we lose two $z$-edges. Case 2: When the overlap just mentioned is a weak fall, we gain two $z$-edges.

Therefore, we have $U(x, y, z)=G_{s q+}\left(x, z^{-2}, z^{2}, y z\right)$.

Next, every piece of a brick wall has something like a twin; the twin is a column-convex polyomino on the honeycomb lattice. See Fig. 12 As regards the three perimeter counts ( $x$-edges, $y$-edges, $z$-edges), between the twins there is no difference. 


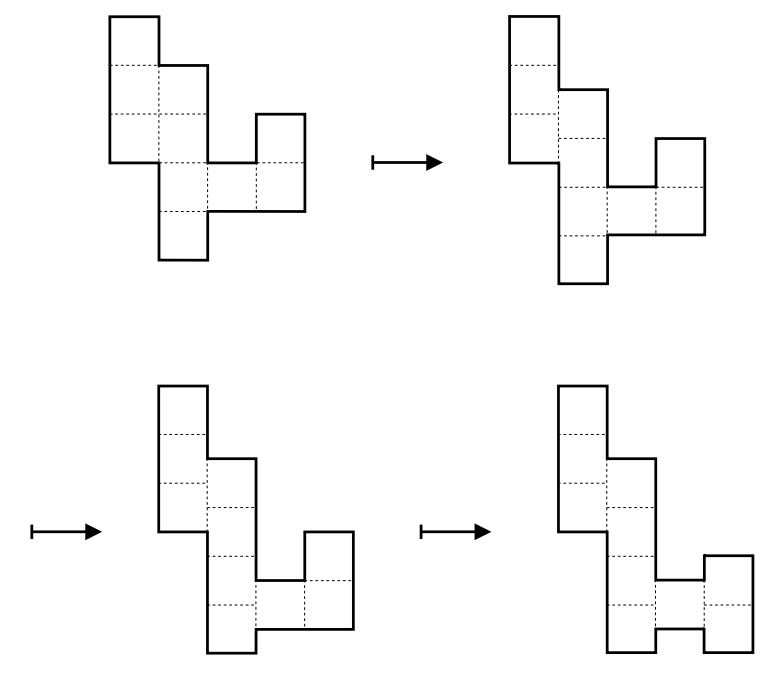

Fig. 10: A column-convex polyomino becomes similar to a piece of a brick wall.

Note that the twin which lives on the honeycomb lattice is subject to one (and only one) restriction on its freedom. Namely, it can have no ceiling overlaps. The definition follows.

Let $P$ be a column-convex polyomino on the honeycomb lattice. A ceiling overlap is the event that, in a pair of adjacent columns of $P$, the bottom cell of the right column is the upper right neighbour of the top cell of the left column.

So, $U(x, y, z)$ is also the perimeter generating function for column-convex polyominoes which live on the honeycomb lattice, but have no ceiling overlaps.

From now on, when we say "a column-convex polyomino", we mean a column-convex polyomino on the honeycomb lattice.

Let $P$ be a column-convex polyomino, and let $P$ have one ceiling overlap. Then we can think of $P$ as two column-convex polyominoes, say $P_{1}$ and $P_{2}$, staying stuck together. Here, neither of $P_{1}$ and $P_{2}$ has any ceiling overlaps. See Fig. 13. Notice that $P$ has two $z$-edges less than $P_{1}$ and $P_{2}$ taken cumulatively.

We now see how to express the perimeter generating functions. For column-convex polyominoes with one ceiling overlap, the expression is $z^{-2} U^{2}$. More generally, $G_{h e x}$-that is, the perimeter generating function for all column-convex polyominoes - can be written as

$$
\begin{aligned}
G_{\text {hex }} & =U+U z^{-2} U+U z^{-2} U z^{-2} U+\ldots \\
& =U\left(1+z^{-2} U+z^{-2} U z^{-2} U+\ldots\right) \\
& =\frac{U}{1-z^{-2} U} .
\end{aligned}
$$

Therefore $U=\frac{G_{h e x}}{1+z^{-2} G_{h e x}}$. Let

$$
\mu_{-}=-\frac{\left(1-y^{2}\right)\left(1-z^{2}\right)}{2\left(1-y^{2} z^{2}\right)}, \quad \mu_{+}=\frac{\left(1+y^{2}\right)\left(1+z^{2}\right)}{2\left(1-y^{2} z^{2}\right)}
$$




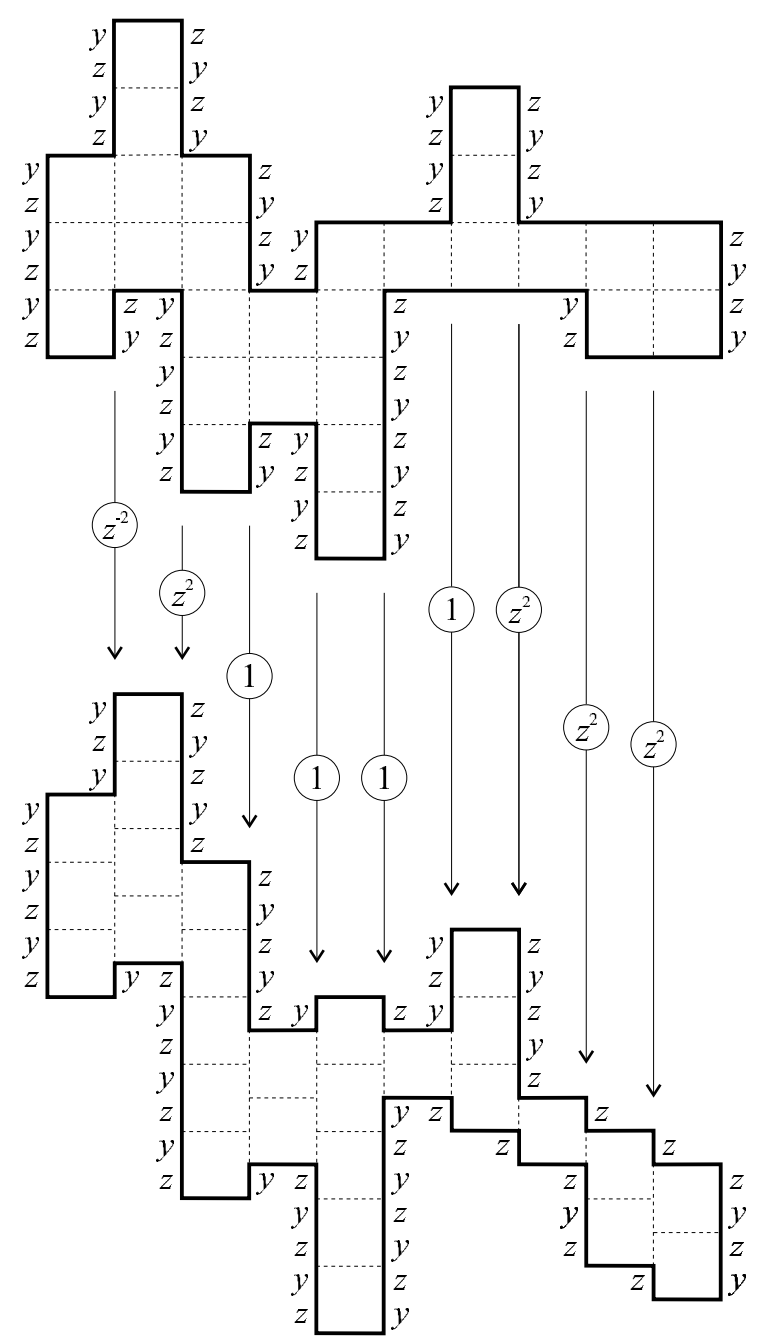

Fig. 11: Effects of the shifts on the vertical perimeter. At every column-column interface there is an arrow, labelled with the local balance of vertical half-edges. 


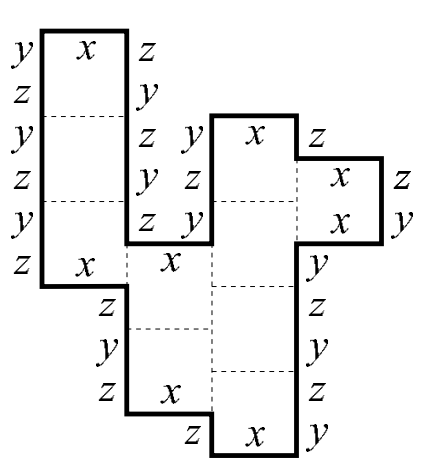

$$
x^{8} y^{12} z^{14} \text { to } U
$$

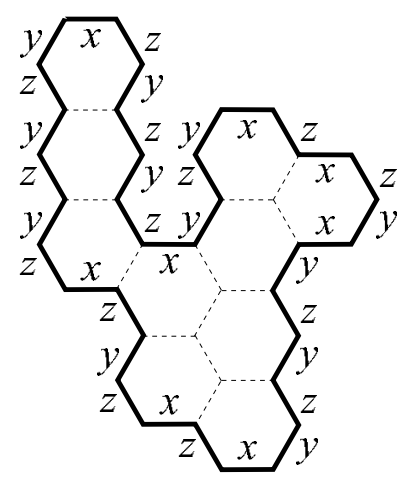

$x^{8} y^{12} z^{14}$ to $U$

Fig. 12: Twin polyominoes.

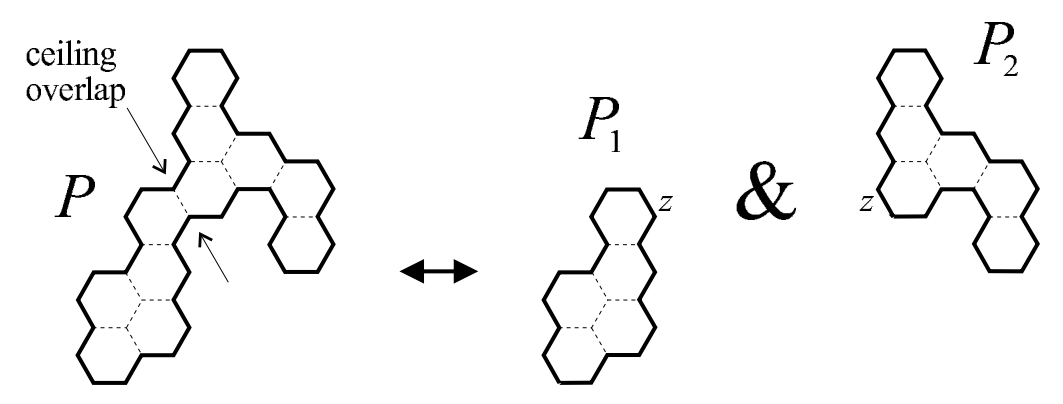

Fig. 13: The ceiling overlap divides $P$ into $P_{1}$ and $P_{2}$. 
and

$$
V=N\left(\frac{x^{2}}{1+x^{2} \mu_{-}^{2}}, 1+\mu_{-}, y z\right) .
$$

Recalling that $U(x, y, z)=G_{s q+}\left(x, z^{-2}, z^{2}, y z\right)$, we now go back to relation (24) and we substitute $z^{-2}$ for $a, z^{2}$ for $b_{0}$, and $y z$ for $y$. We quickly find that

$$
\frac{\left(1-y^{2} z^{2}\right) U}{1-y^{2} z^{2}-\left(z^{-2}+z^{2}-1\right) U}=V,
$$

which, together with the relation between $U$ and $G_{h e x}$, gives

$$
G_{\text {hex }}=\frac{1-y^{2} z^{2}}{1-y^{2}-z^{2}}\left[\frac{2}{1+y^{2}+z^{2}+\left(1-y^{2}-z^{2}\right)\left(1-\frac{2 V}{1-y^{2} z^{2}}\right)}-1\right] .
$$

Next we combine (26) with $[16)$, obtaining

$$
\left(1+x^{2} \mu_{-}^{2}\right)\left(1-\frac{2 V}{1-y^{2} z^{2}}\right)^{4}-\left(1+x^{2}+2 x^{2} \mu_{-} \mu_{+}\right)\left(1-\frac{2 V}{1-y^{2} z^{2}}\right)^{2}+x^{2} \mu_{+}^{2}=0
$$

where $\mu_{-}$and $\mu_{+}$are given by (25).

Now follows the long and tiring, but unavoidable task of solving equation (28). Once we are finished, we embed the formula for $1-\frac{2 V}{1-y^{2} z^{2}}$ into 27). This yields the following theorem.

Theorem 3 The perimeter generating function for column-convex polyominoes on the honeycomb lattice is given by

$$
G_{h e x}(x, y, z)=\frac{1-y^{2} z^{2}}{1-y^{2}-z^{2}} \cdot\left\{\frac{2}{1+y^{2}+z^{2}+\left(1-y^{2}-z^{2}\right) \sqrt{\frac{2+x^{2}\left[1+\frac{\left(y^{2}-z^{2}\right)^{2}}{\left(1-y^{2} z^{2}\right)^{2}}\right]+2 \sqrt{\Delta}}{4+x^{2} \frac{\left(1-y^{2}\right)^{2}\left(1-z^{2}\right)^{2}}{\left(1-y^{2} z^{2}\right)^{2}}}}-1}\right\},
$$

where

$$
\Delta=1-2 x^{2} \frac{y^{2}\left(1+z^{2}\right)^{2}+\left(1+y^{2}\right)^{2} z^{2}}{\left(1-y^{2} z^{2}\right)^{2}}+x^{4} \frac{\left(y^{2}-z^{2}\right)^{2}}{\left(1-y^{2} z^{2}\right)^{2}} .
$$

If we set $y=z$, the formula for $G_{h e x}$ becomes much simpler.

Corollary 1 We have

$$
G_{\text {hex }}(x, y, y)=\frac{1-y^{4}}{1-2 y^{2}}\left[\frac{2}{1+2 y^{2}+\left(1-2 y^{2}\right) \sqrt{\frac{2+x^{2}+2 \sqrt{\delta}}{4+x^{2}\left(\frac{1-y^{2}}{1+y^{2}}\right)^{2}}}}-1\right]
$$


where

$$
\delta=1-\frac{4 x^{2} y^{2}}{\left(1-y^{2}\right)^{2}} .
$$

In the case of the generating function $G_{s q}(x, y)$, the two square roots can be written in two ways: separately, as in (10), and one inside the other, as in (15). However, I think that the formulae for $G_{h e x}(x, y, z)$ and $G_{h e x}(x, y, y)$ cannot be written in a form such that two square roots of rational functions stand separately.

In Maple, there is a command (called mtaylor) for computing multivariate Taylor series. Now, with our formula for $G_{h e x}(x, y, z)$, mtaylor works all right. By contrast, with Lin and Wu's [11] formula for $G_{\text {hex }}(x, y, z)$, mtaylor arrives at an impasse. This happens because the denominator of their formula involves a factor $D=y^{2}+z^{2}+\ldots$, which is a polynomial with no constant term.

\section{Appendix}

A referee has rightly remarked that we should prove (and not just believe in) the equivalence between the formulae for $G_{h e x}$ given in this paper and in Lin and Wu's paper [11]. Hence, we started from Lin and Wu's formula, which reads

$$
G_{h e x}(x, y, z)=\frac{1-y^{2} z^{2}}{4 D}\left[a+b \sqrt{S}+\frac{(c+d \sqrt{S}) \sqrt{T}}{2\left(1+y^{2} z^{2}\right)+x^{2}\left(1-y^{2}\right)\left(1-z^{2}\right)}\right]
$$

where

$$
\begin{aligned}
& a=1+4\left(y^{2}+z^{2}\right)+3 y^{4}+4 y^{2} z^{2}+3 z^{4}-8 y^{2} z^{2}\left(y^{2}+z^{2}\right) \\
& -6 y^{2} z^{2}\left(y^{4}+z^{4}\right)-11 y^{4} z^{4}+4 y^{4} z^{4}\left(y^{2}+z^{2}\right)+3 y^{4} z^{4}\left(y^{2}+z^{2}\right)^{2} \\
& +x^{2}\left[y^{2}+z^{2}-4 y^{2} z^{2}\left(y^{2}+z^{2}\right)-y^{6}-z^{6}\right. \\
& \left.+y^{2} z^{2}\left(y^{6}+z^{6}\right)+5 y^{4} z^{4}\left(y^{2}+z^{2}\right)\right] \text {, } \\
& b=\left(1-y^{2} z^{2}\right)\left[1-\left(y^{2}+z^{2}\right)^{2}\right], \\
& c=-\left(1-y^{2} z^{2}\right)\left[1+4\left(y^{2}+z^{2}\right)+3 y^{4}+7 y^{2} z^{2}+3 z^{4}+4 y^{2} z^{2}\left(y^{2}+z^{2}\right)\right. \\
& \left.+y^{2} z^{2}\left(y^{2}+z^{2}\right)^{2}+x^{2}\left(y^{2}+z^{2}\right)\left(1-y^{4}-z^{4}\right)\right], \\
& d=-1+y^{4}+y^{2} z^{2}+z^{4}-y^{2} z^{2}\left(y^{2}+z^{2}\right)^{2}, \\
& D=\left(y^{2}+z^{2}\right)\left[1+2\left(y^{2}+z^{2}\right)+y^{4}+z^{4}-4 y^{2} z^{2}\left(y^{2}+z^{2}\right)\right. \\
& \left.-y^{2} z^{2}\left(2 y^{4}+3 y^{2} z^{2}+2 z^{4}\right)+2 y^{4} z^{4}\left(y^{2}+z^{2}\right)+y^{4} z^{4}\left(y^{2}+z^{2}\right)^{2}\right] \\
& +x^{2} y^{2} z^{2}\left[1-\left(y^{2}+z^{2}\right)^{2}\left(1-y^{2} z^{2}\right)\right] \text {, } \\
& S=1-2\left(x^{2} y^{2}+y^{2} z^{2}+x^{2} z^{2}\right)-8 x^{2} y^{2} z^{2}+x^{4} y^{4}+y^{4} z^{4}+x^{4} z^{4} \\
& -2 x^{2} y^{2} z^{2}\left(x^{2}+y^{2}+z^{2}\right), \\
& T=2\left(1-y^{2} z^{2}\right)^{2}+2 x^{2}\left[2-\left(y^{2}+z^{2}\right)\left(1+y^{2} z^{2}\right)+2 y^{4} z^{4}\right] \\
& +x^{4}\left(1-y^{2}\right)^{2}\left(1-z^{2}\right)^{2}+2\left(1-y^{2} z^{2}\right) \sqrt{S} .
\end{aligned}
$$


By appropriately squaring two times, from (30) we got a degree-four algebraic equation. We factored that equation (at the price of employing pretty much RAM), and we removed the redundant factors. The result was a sensibly shorter degree-four algebraic equation, still satisfied by Lin and Wu's expression (30). Let this latter degree-four algebraic equation be numbered (A). Next, we wanted to know whether equation (A) is satisfied by our expression (29), too. Much to our relief, the answer was yes. However, equation (A) has four solutions. To show that (29) and (30) are one and the same solution of (A), we computed all four solutions of (A). In the case $x=y=z$, those four solutions have power series expansions

$$
\begin{gathered}
1-x-\frac{3}{2} x^{2}+\ldots, \quad 1+x-\frac{3}{2} x^{2}+\ldots, \\
x^{6}+3 x^{10}+2 x^{12}+\ldots \quad \text { and } \quad \frac{1}{2} x^{-2}-\frac{3}{4} x^{2}-\frac{5}{8} x^{6}+\ldots .
\end{gathered}
$$

Now, the power series expansions of (29) and (30) both begin with a sixth-order term. (This must be so. On the hexagonal lattice, the one-celled polyomino has perimeter 6.) Hence, (29) and (30) are both equal to that solution of (A) which gave us the series $x^{6}+3 x^{10}+2 x^{12}+\ldots$ That is to say, our expression 29) and Lin and Wu's expression (30) are equal to one another.

\section{Acknowledgements}

I would like to thank the referees for several helpful comments, and Professor Dragutin Svrtan both for further comments and for kind help in computer matters.

\section{References}

[1] M. Bousquet-Mélou, New enumerative results on two-dimensional directed animals, Discrete Math. 180 (1998), 73-106.

[2] M. Bousquet-Mélou, A. Rechnitzer, Lattice animals and heaps of dimers, Discrete Math. 258 (2002), 235-274.

[3] R. Brak, A.J. Guttmann, I.G. Enting, Exact solution of the row-convex polygon perimeter generating function, J. Phys. A: Math. Gen. 23 (1990), 2319-2326.

[4] M.P. Delest, Generating functions for column-convex polyominoes, J. Combin. Theory Ser. A 48 (1988), 12-31.

[5] D. Dhar, M.K. Phani, M. Barma, Enumeration of directed site animals on two-dimensional lattices, J. Phys. A: Math. Gen. 15 (1982), L279-L284.

[6] S. Feretić, The column-convex polyominoes perimeter generating function for everybody, Croat. Chem. Acta 69 (1996), 741-756.

[7] S. Feretić, A new way of counting the column-convex polyominoes by perimeter, Discrete Math. 180 (1998), 173-184. 
[8] S. Feretić, D. Svrtan, On the number of column-convex polyominoes with given perimeter and number of columns, in: A. Barlotti, M. Delest, R. Pinzani (Eds.), Proc. Fifth FPSAC Conf., Firenze, 1993, pp. 201-214.

[9] D.A. Klarner, Cell growth problems, Canad. J. Math. 19 (1967), 851-863.

[10] K.Y. Lin, Perimeter generating function for row-convex polygons on the rectangular lattice, J. Phys. A: Math. Gen. 23 (1990), 4703-4705.

[11] K.Y. Lin, F.Y. Wu, Unidirectional-convex polygons on the honeycomb lattice, J. Phys. A: Math. Gen. 23 (1990), 5003-5010.

[12] J.-G. Penaud, Arbres et animaux, Mémoire présenté en vue de l'habilitation à diriger les recherches, Université Bordeaux 1, 1990.

[13] G.-C. Rota, Report on the present state of combinatorics, Discrete Math. 153 (1996), 289-303.

[14] L.W. Shapiro, Directed animals and Motzkin paths, informal communication, 1999.

[15] H.N.V. Temperley, Combinatorial problems suggested by the statistical mechanics of domains and of rubber-like molecules, Phys. Rev. 103 (1956), 1-16. 
